\title{
Global Asymptotic Stability of Stochastic Nonautonomous Lotka-Volterra Models with Infinite Delay
}

\author{
Fengying Wei and Yuhua Cai \\ College of Mathematics and Computer Science, Fuzhou University, Fuzhou 350116, China \\ Correspondence should be addressed to Fengying Wei; weifengying@fzu.edu.cn
}

Received 1 July 2013; Revised 4 November 2013; Accepted 4 November 2013

Academic Editor: Julio Rossi

Copyright (C) 2013 F. Wei and Y. Cai. This is an open access article distributed under the Creative Commons Attribution License, which permits unrestricted use, distribution, and reproduction in any medium, provided the original work is properly cited.

\begin{abstract}
A kind of general stochastic nonautonomous Lotka-Volterra models with infinite delay is investigated in this paper. By constructing several suitable Lyapunov functions, the existence and uniqueness of global positive solution and global asymptotic stability are obtained. Further, the solution asymptotically follows a normal distribution by means of linearizing stochastic differential equation. Moment estimations in time average are derived to improve the approximation distribution. Finally, numerical simulations are given to illustrate our conclusions.
\end{abstract}

\section{Introduction}

The impact of random factors cannot be neglected in the real world. Different kinds of random perturbations of stochastic models have been investigated in many pieces of literature. Bahar and Mao [1] discussed a stochastic delay Lotka-Volterra model, and they showed that environmental noise would suppress a potential population explosion and also made the solutions to be stochastically ultimately bounded. Almost at the same time, Mao [2] revealed that different types of environmental noise had different effects on delay population models. Meanwhile, Jiang and Shi [3] considered a randomized nonautonomous Logistic equation and represented the unique continuous global positive solution and positive $T$ periodic solution.

Recently, stochastic models with delay are paid more attention by many researchers. Shen et al. [4] studied stochastic Lotka-Volterra competitive models with variable delay, and they obtained the unique global positive solution, stochastically ultimate boundedness, and moment average in time of the solutions. Liu and Wang [5] considered stochastic Lotka-Volterra models with infinite delay; they replaced the intrinsic growth rate by a random perturbation term which was dependent on the difference between the population size and the equilibrium state in their model, and then the sufficient criteria for global asymptotic stability of the solution were established. Huang et al. [6] extended the conclusion of Liu and Wang [5] to a general case. Based on a general phase space

$$
\begin{aligned}
C_{r}:=\left\{\varphi \in C\left((-\infty, 0] ; R_{++}^{n}\right):\right. \\
\left.\quad\|\varphi\|_{C_{r}}=\sup _{-\infty<s \leq 0} e^{r s}|\varphi(s)|<\infty\right\}, \quad r>0,
\end{aligned}
$$

$\mathrm{Xu}$ et al. [7] and $\mathrm{Xu}$ [8] investigated an autonomous stochastic Lotka-Volterra model with infinite delay

$$
\begin{aligned}
d x(t)= & \operatorname{diag}\left(x_{1}(t), \ldots, x_{n}(t)\right) \\
\times & {\left[\left(b+A x(t)+B \int_{-\infty}^{0} x(t+\theta) d \mu(\theta)\right) d t\right.} \\
& +\sigma x(t) d B(t)] ;
\end{aligned}
$$

they established the asymptotic pathwise properties of the solution to model (1), where $x=\left(x_{1}, \ldots, x_{n}\right)^{T}, b=$ $\left(b_{1}, \ldots, b_{n}\right)^{T}, A=\left(a_{i j}\right)_{n \times n}, B=\left(b_{i j}\right)_{n \times n}$, and $\sigma=\left(\sigma_{i j}\right)_{n \times n} ; C_{r}$ was a Banach space (see $[7,9,10]) ; \mu$ was probability measure defined on $(-\infty, 0]$ such that

$$
\mu_{r}:=\int_{-\infty}^{0} e^{-2 r \theta} d \mu(\theta)<\infty, \quad r>0 .
$$


In particular, when $\mu(\theta)=e^{k r \theta}(k>2)$ for $\theta \leq 0$, assumption (H2) was satisfied.

Motivated by the works mentioned previously, we always assume that the intensity of white noise is dependent on the difference between the population size and the equilibrium state and also is dependent on time $t$ in this paper. Now, we consider a more general stochastic nonautonomous LotkaVolterra model with infinite delay

$$
\begin{aligned}
d x_{i}(t)=x_{i}(t)\left[h_{i}(t)+\sum_{j=1}^{n} a_{i j}(t) x_{j}(t)\right. & \\
& +\sum_{j=1}^{n} b_{i j}(t) x_{j}(t-\delta(t)) \\
& \left.+\sum_{j=1}^{n} c_{i j}(t) \int_{-\infty}^{0} x_{j}(t+\theta) d \mu(\theta)\right] d t \\
+x_{i}(t) & {\left[\sigma_{i}(t)\left(x_{i}(t)-x_{i}^{*}\right)\right.} \\
& +\sum_{j=1}^{n} \alpha_{i j}(t) x_{j}(t)\left(x_{i}(t)-x_{i}^{*}\right) \\
& +\sum_{j=1}^{n} \beta_{i j}(t) x_{j}(t-\delta(t))\left(x_{i}(t)-x_{i}^{*}\right) \\
& \left.+\sum_{j=1}^{n} \gamma_{i j}(t)\right]_{-\infty}^{0} x_{j}(t+\theta) d \mu(\theta) \\
& \left.\times\left(x_{i}(t)-x_{i}^{*}\right)\right] d B_{i}(t), i=1,2, \ldots, n,
\end{aligned}
$$

where $h_{i}(t)>0, a_{i j}(t)\left(a_{i i}(t)<0\right.$ if $\left.i=j\right), b_{i j}(t)$ and $c_{i j}(t)$ are parameter functions; noise intensities $\sigma_{i}(t)>0, \alpha_{i j}(t) \geq 0$, $\beta_{i j}(t) \geq 0$ and $\gamma_{i j}(t) \geq 0$ are continuous bounded function on $[0,+\infty)$; variable delay function $\delta:[0, \infty) \rightarrow[0, \tau]$ and $\delta^{\prime}(t) \leq 0 ; x^{*}=\left(x_{1}^{*}, x_{2}^{*}, \ldots, x_{n}^{*}\right)^{T}$ denotes an equilibrium state with respect to the deterministic part of model (2) in $R_{++}^{n}$ (we always assume that such $x^{*}$ exists in this paper). For simplicity, model (2) can be rewritten in the following form:

$$
d x_{i}(t)=x_{i}(t) f_{i} d t+x_{i}(t) g_{i} d B_{i}(t), \quad i=1,2, \ldots, n .
$$

Next, we will investigate the global positive solution and its asymptotic properties of model (2). Further, the approximation distribution of solutions to model (2) is explored. Our results will extend some classical deterministic results into the stochastic cases.

\section{Preliminary}

Let $\left(\Omega, \mathscr{F},\left\{\mathscr{F}_{t}\right\}_{t \geq 0}, P\right)$, throughout this paper unless otherwise specified, be a complete probability space with a filtration $\left\{\mathscr{F}_{t}\right\}_{t \geq 0}$ satisfying the usual conditions, and
$B_{i}(t)(i=1,2, \ldots, n)$ denote the independent 1-dimensional standard Brownian motion defined on the complete probability space. We denote the nonnegative cone and positive cone by $R_{+}^{n}, R_{++}^{n}$, respectively; that is, $R_{+}^{n}=\left\{x \in R^{n}: x_{i} \geq\right.$ $0, i=1,2, \ldots, n\}, R_{++}^{n}=\left\{x \in R^{n}: x_{i}>0, i=1,2, \ldots, n\right\}$. If $x \in R^{n}$, its norm is denoted by $|x|=\left(\sum_{i=1}^{n} x_{i}^{2}\right)^{1 / 2}$; if $A$ is a vector or matrix, its transpose is denoted by $A^{T}$; if $A$ is matrix, trace norm of matrix $A$ is denoted by $|A|=\sqrt{\operatorname{trace}\left(A^{T} A\right)}$; if $A$ is negative matrix, we denote it by $A<0$. Suppose that $y(t)$ is a continuous bounded function on $[0,+\infty)$; we define $y_{u}=\max _{t \in[0,+\infty)} y(t), y_{l}=\min _{t \in[0,+\infty)} y(t)$, with usual assumption $\inf \emptyset=\infty$, where $\emptyset$ denotes the empty set.

\section{The Existence and Uniqueness of Global Positive Solution}

In this section, we show that model (2) has a unique global solution, and the solution will remain in $R_{++}^{n}$ with probability 1 .

Theorem 1. If (H1) and (H2) hold, then there is a unique solution $x(t)$ to model (2). Moreover, $x(t)$ remains in $R_{++}^{n}$ with probability 1 , where $t \in R$.

Proof. Clearly, the coefficients of model (2) satisfy local Lipschitz continuous but do not satisfy the linear growth condition. To show that the solution $x(t)$ is global, a.s., $\tau_{e}=$ $\infty$ is needed now, where $\tau_{e}$ is the explosion time.

Let $k_{0}>0$ be sufficiently large such that each component of initial data $\xi(0)$ is lying in the interval $\left(1 / k_{0}, k_{0}\right)$. For each integer $k \geq k_{0}$, define the stopping time

$$
\begin{array}{r}
\tau_{k}=\inf \left\{t \in\left[0, \tau_{e}\right): x_{i} \notin\left(\frac{1}{k}, k\right),\right. \\
\text { for some } i=1,2, \ldots, n\} .
\end{array}
$$

Clearly, $\tau_{k}$ is increasing as $k \rightarrow \infty$. Set $\tau_{\infty}=\lim _{k \rightarrow \infty} \tau_{k}$; hence, $\tau_{\infty} \leq \tau_{e}$ a.s.. If we can prove that $\tau_{\infty}=\infty$ a.s., then $\tau_{e}=$ $\infty$ a.s.. To prove this statement, let us define a $C^{2}$-function $V_{1}: R_{++}^{n} \rightarrow R_{++}$by $V_{1}(x)=\sum_{i=1}^{n} x_{i}^{p}-p \log x_{i}$, where $p \epsilon$ $(0,1)$. It is easy to see that $V_{1}(x)>0$ for all $x \in R_{++}^{n}$. Applying the Itô formula to model (2), it leads to

$$
d V_{1}(x(t))=L V_{1}(x(t)) d t+\sum_{i=1}^{n} p\left(x_{i}^{p}(t)-1\right) g_{i} d B_{i}(t),
$$

where

$$
L V_{1}(x)=p \sum_{i=1}^{n}\left(x_{i}^{p}-1\right) f_{i}+\frac{1}{2} p \sum_{i=1}^{n}\left((p-1) x_{i}^{p}+1\right) g_{i}^{2}
$$

From the elementary inequality $a b \leq(1 / 2)\left(a^{2}+b^{2}\right)$ and Hölder's inequality, we have

$$
\begin{aligned}
\sum_{i=1}^{n}\left(x_{i}^{p}-1\right) f_{i} \leq & \sum_{i=1}^{n} h_{i u}\left(x_{i}^{p}+1\right)+\frac{1}{2} \sum_{i=1}^{n} \sum_{j=1}^{n}\left|a_{i j}\right|_{u}\left(x_{i}^{p}-1\right)^{2} \\
& +\frac{1}{2} \sum_{i=1}^{n} \sum_{j=1}^{n}\left|a_{i j}\right|_{\mathcal{u}} x_{j}^{2}+\frac{1}{2} \sum_{i=1}^{n} \sum_{j=1}^{n}\left|b_{i j}\right|_{u}^{2}\left(x_{i}^{p}-1\right)^{2}
\end{aligned}
$$




$$
\begin{aligned}
& +\frac{1}{2} \sum_{i=1}^{n} \sum_{j=1}^{n} x_{j}^{2}(t-\delta(t)) \\
& +\frac{1}{2} \sum_{i=1}^{n} \sum_{j=1}^{n}\left|c_{i j}\right|_{u}^{2}\left(x_{i}^{p}-1\right)^{2} \\
& +\frac{1}{2} \sum_{i=1}^{n} \sum_{j=1}^{n} \int_{-\infty}^{0} x_{j}^{2}(t+\theta) d \mu(\theta) .
\end{aligned}
$$

By the fact that $K=\left(\|\xi\|_{C_{r}}+\|x\|_{C_{r}}\right)^{2}<\infty, u^{2} / \rho_{j}-v^{2} /\left(\rho_{j}-\right.$ $1) \leq(u+v)^{2} \leq u^{2} / l_{i}+v^{2} /\left(1-l_{i}\right), 0<l_{i}<1<\rho_{j}$, and $\left(\sum_{i=1}^{n} a_{i} b_{i}\right)^{2} \leq \sum_{i=1}^{n} a_{i}^{2} \sum_{i=1}^{n} b_{i}^{2}$, it implies that

$$
\begin{aligned}
& g_{i}^{2} \leq\left(x_{i}-x_{i}^{*}\right)^{2}\left\{\frac{1}{l_{1}} \sigma_{i u}^{2}+\frac{1}{l_{2}\left(1-l_{1}\right)}\right. \\
& \times \sum_{j=1}^{n}\left|\alpha_{i j}\right|_{u}^{2} \sum_{j=1}^{n} x_{j}^{2} \\
& +\frac{1}{l_{3}\left(1-l_{2}\right)\left(1-l_{1}\right)} \sum_{j=1}^{n}\left|\beta_{i j}\right|_{u}^{2} \\
& \times \sum_{j=1}^{n} x_{j}^{2}(t-\delta(t)) \\
& +\frac{1}{\left(1-l_{3}\right)\left(1-l_{2}\right)\left(1-l_{1}\right)} \sum_{j=1}^{n}\left|\gamma_{i j}\right|_{u}^{2} \\
& \left.\times \sum_{j=1}^{n} \int_{-\infty}^{0} x_{j}^{2}(t+\theta) d \mu(\theta)\right\} \\
& \leq\left(x_{i}-x_{i}^{*}\right)^{2}\left\{\frac{1}{l_{1}} \sigma_{i u}^{2}+\frac{K}{l_{2}\left(1-l_{1}\right)} \sum_{j=1}^{n}\left|\alpha_{i j}\right|_{u}^{2}\right. \\
& +\frac{K}{l_{3}\left(1-l_{2}\right)\left(1-l_{1}\right)} \sum_{j=1}^{n}\left|\beta_{i j}\right|_{u}^{2} \\
& \left.+\frac{K}{\left(1-l_{3}\right)\left(1-l_{2}\right)\left(1-l_{1}\right)} \sum_{j=1}^{n}\left|\gamma_{i j}\right|_{u}^{2}\right\} \\
& :=K_{1}\left(x_{i}-x_{i}^{*}\right)^{2} \text {, } \\
& g_{i}^{2} \geq\left(x_{i}-x_{i}^{*}\right)^{2}\left\{\sigma_{i l}^{2}+\left(\sum_{j=1}^{n} \alpha_{i j}(t) x_{j}\right)^{2}\right. \\
& +\left(\sum_{j=1}^{n} \beta_{i j}(t) x_{j}(t-\delta(t))\right)^{2}
\end{aligned}
$$

$$
\begin{aligned}
\left.+\left(\sum_{j=1}^{n} \gamma_{i j}(t) \int_{-\infty}^{0} x_{j}^{2}(t+\theta) d \mu(\theta)\right)^{2}\right\} \\
\geq \sigma_{i l}^{2}\left(x_{i}-x_{i}^{*}\right)^{2}:=K_{2}\left(x_{i}-x_{i}^{*}\right)^{2},
\end{aligned}
$$

where $0<K_{2}<K_{1}$ for any $l_{i} \in(0,1)$. Then (6) yields that

$$
\begin{aligned}
L V_{1}(x) \leq \sum_{i=1}^{n}\left\{p h_{i u}\left(x_{i}^{p}+1\right)\right. & \\
& +\frac{p}{2} \sum_{j=1}^{n}\left(\left|a_{i j}\right|_{u}+\left|b_{i j}\right|_{u}^{2}+\left|c_{i j}\right|_{u}^{2}\right)\left(x_{i}^{p}-1\right)^{2} \\
& +\frac{p}{2} \sum_{j=1}^{n}\left|a_{i j}\right|_{u} x_{j}^{2}+\frac{p K_{1}}{2}\left(x_{i}-x_{i}^{*}\right)^{2} \\
& \left.-\frac{p(1-p) K_{2}}{2} x_{i}^{p}\left(x_{i}-x_{i}^{*}\right)^{2}\right\} \\
+ & \frac{p}{2} \sum_{i=1}^{n} \sum_{j=1}^{n} x_{j}^{2}(t-\delta(t)) \\
+ & \frac{p}{2} \sum_{i=1}^{n} \sum_{j=1}^{n} \int_{-\infty}^{0} x_{j}^{2}(t+\theta) d \mu(\theta)
\end{aligned}
$$

Define $V_{2}(x(t))=V_{1}(x(t))+(p / 2) \sum_{i=1}^{n} \sum_{j=1}^{n} \int_{t-\delta(t)}^{t} x_{j}^{2}(s) d s+$ $(p / 2) \sum_{i=1}^{n} \sum_{j=1}^{n} \int_{-\infty}^{0} \int_{t+\theta}^{t} x_{j}^{2}(s) d s d \mu(\theta)$; the proof is easily checked (details can be found at the appendix).

\section{Global Asymptotic Stability}

Theorem 2. If $(H 1)$ and $(H 2)$ hold, there exist positive numbers $\bar{d}_{1}, \bar{d}_{2}, \ldots, \bar{d}_{n}$ such that $\overline{D A}+\bar{A}^{T} \bar{D}$ is negative definite, and then

$$
\lim _{t \rightarrow \infty} x_{i}(t)=x_{i}^{*} \quad \text { a.s., } \quad i=1,2, \ldots, n
$$

that is, $x^{*}$ is globally asymptotically stable a.s., where $K=$ $\left(\|\xi\|_{C_{r}}+\|x\|_{C_{r}}\right)^{2}, \bar{D}=\operatorname{diag}\left(\bar{d}_{1}, \bar{d}_{2}, \ldots, \bar{d}_{n}\right), \bar{A}=\left(\eta_{i j}\right)_{n \times n}$, $\eta_{i j}=a_{i j}$ for $i \neq j$, and

$$
\begin{aligned}
\eta_{i i}=\left(a_{i i}\right)_{u}+0.5 x_{i}^{*}\left[\sigma_{i u}^{2}+K\left|\sum_{j=1}^{n} \alpha_{i j}\right|_{u}^{2}\right. \\
\left.+K\left|\sum_{j=1}^{n} \beta_{i j}\right|_{u}^{2}+K\left|\sum_{j=1}^{n} \gamma_{i j}\right|_{u}^{2}\right]
\end{aligned}
$$




$$
\begin{aligned}
+0.5\left[\sum_{j=1}^{n}\left|b_{i j}\right|_{u}+\sum_{j=1}^{n}\left|c_{i j}\right|_{u}\right. \\
\left.+\sum_{j=1}^{n} \frac{\bar{d}_{j}}{\bar{d}_{i}}\left|b_{j i}\right|_{u}+\sum_{j=1}^{n} \frac{\bar{d}_{j}}{\bar{d}_{i}}\left|c_{j i}\right|_{u}\right] .
\end{aligned}
$$

Proof. Define a $C^{2}$-function $V_{3}: R_{++}^{n} \rightarrow R_{++}$by $V_{3}(x)=$ $\sum_{i=1}^{n} \bar{d}_{i}\left(x_{i}-x_{i}^{*}-x_{i}^{*} \log \left(x_{i} / x_{i}^{*}\right)\right)$. Similar to the proof of [6] (see Theorem 2.1), we can derive

$$
\begin{aligned}
d V_{3}(x(t)) \leq & L V_{3}(x(t)) d t+\sum_{i=1}^{n} \bar{d}_{i}\left(x_{i}(t)-x_{i}^{*}\right) g_{i} d B_{i}(t) \\
\leq & \frac{1}{2}\left(x_{i}(t)-x_{i}^{*}\right)\left(\overline{D A}+\bar{A}^{T} \bar{D}\right)\left(x_{i}(t)-x_{i}^{*}\right)^{T} d t \\
& +\sum_{i=1}^{n} \bar{d}_{i}\left(x_{i}(t)-x_{i}^{*}\right) g_{i} d B_{i}(t) .
\end{aligned}
$$

Since $\overline{D A}+\bar{A}^{T} \bar{D}$ is negative definite, $L V_{3}(x)<0$ is valid along trajectories in $R_{++}^{n}$ except $x^{*}$. The proof is complete.

\section{Approximation Distribution of Solution}

Theorem 3. If (H1), (H2), and the conditions of Theorem 2 hold, then each component $x_{i}(t)$ of solution $x(t)$ to model (2) follows asymptotically 1-dimensional normal distribution $\mathcal{N}\left(x_{i}^{*},+\infty\right)$, where $i=1,2, \ldots, n$.

Proof. By the definition of equilibrium state $x_{i}^{*} \neq 0$, then $f_{i}\left(x_{i}^{*}\right)=0$. Since $x^{*}$ is stable for the deterministic part to model (2), then $\left.\partial_{x_{i}}\left(f_{i}(x) x_{i}\right)\right|_{x_{i}=x_{i}^{*}}:=m_{i}<0$ for $i=1,2, \ldots, n$.

Linearizing the $i$ th equation of model (2) by Taylor expansion at $x_{i}^{*}$, then we have

$$
\begin{aligned}
d\left(x_{i}-x_{i}^{*}\right) \approx[ & f_{i}\left(x_{i}^{*}\right) x_{i}^{*}+\left.\partial_{x_{i}}\left(f_{i}(x) x_{i}\right)\right|_{x_{i}=x_{i}^{*}} \\
& \left.\times\left(x_{i}-x_{i}^{*}\right)+o\left(\left(x_{i}-x_{i}^{*}\right)^{2}\right)\right] d t \\
+ & {\left[g_{i}\left(x_{i}^{*}\right) x_{i}^{*}+\left.\partial_{x_{i}}\left(g_{i}(x) x_{i}\right)\right|_{x_{i}=x_{i}^{*}}\right.} \\
& \left.\times\left(x_{i}-x_{i}^{*}\right)+o\left(\left(x_{i}-x_{i}^{*}\right)^{2}\right)\right] d B_{i}(t) .
\end{aligned}
$$

Denote $x_{j}(t-\delta(t))=x_{j \delta}(t)$ and $\int_{-\infty}^{0} x_{j}(t+\theta) d \mu(\theta)=x_{j \theta}(t)$, $j=1,2, \cdots, n$. Since $g_{i}\left(x_{i}^{*}\right)=0$ and $\left.\partial_{x_{i}}\left(g_{i}(x) x_{i}\right)\right|_{x_{i}=x_{i}^{*}}=$ $\sigma_{i} x_{i}^{*}+\left.x_{i}^{*} \sum_{j=1}^{n}\left(\alpha_{i j} x_{j}+\beta_{i j} x_{j \delta}+\gamma_{i j} x_{j \theta}\right)\right|_{x_{i}=x_{i}^{*}}:=u_{i} \neq 0$, (13) can be simplified as

$$
\begin{aligned}
\frac{d\left(x_{i}-x_{i}^{*}\right)}{d t} \approx & m_{i}\left(x_{i}-x_{i}^{*}\right) \\
& +u_{i}\left(x_{i}-x_{i}^{*}\right) \frac{d B_{i}(t)}{d t}, \quad i=1,2, \ldots, n,
\end{aligned}
$$

where $m_{i}<0, u_{i} \neq 0$. Then (14) implies that

$$
x_{i}(t)-x_{i}^{*}=\left(x_{i}(0)-x_{i}^{*}\right) e^{m_{i} t+u_{i} B_{i}(t)}, \quad i=1,2, \ldots, n .
$$

From the definition of 1-dimensional Brownian motion, we can derive that $x_{i}(t) \sim \mathcal{N}\left(x_{i}^{*}+\left(x_{i}(0)-x_{i}^{*}\right) e^{m_{i} t}\right.$, $\left.\left(x_{i}(0)-x_{i}^{*}\right)^{2} e^{u_{i}^{2} t}\right)$. According to the conditions of Theorem 2, when $t \rightarrow+\infty$, one can find that $u_{i} \rightarrow u_{i}^{*}=\sigma_{i} x_{i}^{*}+$ $x_{i}^{*} \sum_{j=1}^{n}\left(\alpha_{i j} x_{j}^{*}+\beta_{i j} x_{j \delta}^{*}+\gamma_{i j} x_{j \theta}^{*}\right) \neq 0$ a.s.; thus, $\lim _{t \rightarrow+\infty} x_{i}(t) \sim$ $\mathcal{N}\left(x_{i}^{*}+\infty\right)$; in other words, $x_{i}(t)$ asymptotically follows 1dimensional normal distribution. When $t$ tends to $\infty$, the mean of solution $x(t)$ to model (2) is $x^{*}$; it is just consistent with the conclusion of Theorem 2. However, the deviation between solution $x_{i}(t)$ and mean $x_{i}^{*}$ may approach infinity; it is bad information for further analysis. Now, if the variance can be evaluated, the disadvantage will be improved.

\section{Moment Estimation}

First, let us prove one useful moment estimation.

Theorem 4. If (H1) and (H2) hold, then there is a positive constant $G=G(p)$, such that $\limsup _{t \rightarrow \infty} E|x(t)|^{p} \leq G$, where $p \in(0,1)$.

Proof. Define a $C^{2}$-function $V_{4}: R_{++}^{n} \rightarrow R_{++}$by $V_{4}(x)=$ $\sum_{i=1}^{n} x_{i}^{p}$. For any given $\varepsilon \in(0,2 r)$, applying the Itô formula to $e^{\varepsilon t} V_{4}(x(t))$ and taking expectation, it yields that

$$
\begin{aligned}
e^{\varepsilon t} E V_{4}(x(t))= & E V_{4}(\xi(0)) \\
& +E \int_{0}^{t} e^{\varepsilon s}\left[L V_{4}(x(s))+\varepsilon V_{4}(x(s))\right] d s,
\end{aligned}
$$

where

$$
L V_{4}(x)=p \sum_{i=1}^{n} x_{i}^{p} f_{i}-\frac{p(1-p)}{2} \sum_{i=1}^{n} x_{i}^{p} g_{i}^{2} .
$$

By fundamental inequality $a b \leq(1 / 2)\left(a^{2}+b^{2}\right)$,

$$
\begin{aligned}
& \sum_{i=1}^{n} x_{i}^{p} f_{i} \leq \sum_{i=1}^{n} h_{i u} x_{i}^{p}+\frac{1}{2} \sum_{i=1}^{n} \sum_{j=1}^{n}\left|a_{i j}\right|_{u} x_{i}^{2 p} \\
&+\frac{1}{2} \sum_{i=1}^{n} \sum_{j=1}^{n}\left|a_{i j}\right|_{u} x_{j}^{2}+\frac{1}{2} \sum_{i=1}^{n} \sum_{j=1}^{n}\left|b_{i j}\right|_{u}^{2} x_{i}^{2 p} \\
&+\frac{1}{2} \sum_{i=1}^{n} \sum_{j=1}^{n}\left|c_{i j}\right|_{u}^{2} x_{i}^{2 p} \\
&+\frac{1}{2} \sum_{i=1}^{n} \sum_{j=1}^{n} x_{j}^{2}(s-\delta(s)) \\
&+\frac{1}{2} \sum_{i=1}^{n} \sum_{j=1}^{n} \int_{-\infty}^{0} x_{j}^{2}(s+\theta) d \mu(\theta), \\
& \sum_{i=1}^{n} x_{i}^{p} g_{i}^{2} \geq \sum_{i=1}^{n} K_{2} x_{i}^{p}\left(x_{i}-x_{i}^{*}\right)^{2} .
\end{aligned}
$$


Consequently,

$$
\begin{aligned}
L V_{4}(x(s)) \leq & p \sum_{i=1}^{n} h_{i u} x_{i}^{p} \\
& +\frac{p}{2} \sum_{i=1}^{n} \sum_{j=1}^{n}\left(\left|a_{i j}\right|_{u}+\left|b_{i j}\right|_{u}^{2}+\left|c_{i j}\right|_{u}^{2}\right) x_{i}^{2 p} \\
& +\frac{p}{2} \sum_{i=1}^{n} \sum_{j=1}^{n}\left|a_{i j}\right|_{u} x_{j}^{2} \\
& +\frac{p}{2} \sum_{i=1}^{n} \sum_{j=1}^{n} x_{j}^{2}(s-\delta(s)) \\
& +\frac{1}{2} \sum_{i=1}^{n} \sum_{j=1}^{n} \int_{-\infty}^{0} x_{j}^{2}(s+\theta) d \mu(\theta) \\
& -\frac{p(1-p)}{2} \sum_{i=1}^{n} K_{2} x_{i}^{p}\left(x_{i}-x_{i}^{*}\right)^{2} \\
:= & H(x)-\varepsilon V_{4}(x)+\frac{n p}{2}\left[|x(s-\delta(s))|^{2}-e^{\varepsilon \tau}|x|^{2}\right] \\
& +\frac{n p}{2}\left[\int_{-\infty}^{0}|x(s+\theta)|^{2} d \mu(\theta)-\mu_{r}|x|^{2}\right],
\end{aligned}
$$

where

$$
\begin{aligned}
H(x)= & \sum_{i=1}^{n}\left(p h_{i u}+\varepsilon\right) x_{i}^{p} \\
& +\frac{p}{2} \sum_{i=1}^{n} \sum_{j=1}^{n}\left(\left|a_{i j}\right|_{u}+\left|b_{i j}\right|_{u}^{2}+\left|c_{i j}\right|_{u}^{2}\right) x_{i}^{2 p} \\
& +\frac{n p}{2}\left(e^{\varepsilon \tau}+\mu_{r}\right)|x|^{2} \\
& +\frac{p}{2} \sum_{i=1}^{n} \sum_{j=1}^{n}\left|a_{i j}\right|_{u} x_{j}^{2} \\
& -\frac{p(1-p) K_{2}}{2} \sum_{i=1}^{n} x_{i}^{p}\left(x_{i}-x_{i}^{*}\right)^{2} .
\end{aligned}
$$

Noting that $H(x)$ is bounded in $R_{++}^{n}$; namely, $K_{3}$ := $\sup _{x \in R_{++}^{n}} H(x)<\infty$. Substituting this into (16), it thus follows that

$$
\begin{aligned}
& e^{\varepsilon t} E V_{4}(x(t)) \leq E V_{4}(\xi(0))+\frac{1}{\varepsilon} K_{3}\left(e^{\varepsilon t}-1\right)+\frac{n p}{2} \\
& \times E \int_{0}^{t} e^{\varepsilon s}\left[|x(s-\delta(s))|^{2}-e^{\varepsilon \tau}|x(s)|^{2}\right. \\
&+\int_{-\infty}^{0}|x(s+\theta)|^{2} d \mu(\theta) \\
&\left.-\mu_{r}|x(s)|^{2}\right] d s .
\end{aligned}
$$

By (H1) and (H2), we have

$$
\begin{aligned}
& E \int_{0}^{t} e^{\varepsilon s}\left[|x(s-\delta(s))|^{2}-e^{\varepsilon \tau}|x(s)|^{2}\right] d s \\
& \leq E\left[\int_{-\tau}^{t} e^{\varepsilon(s+\tau)}|x(s)|^{2} d s-\int_{0}^{t} e^{\varepsilon(s+\tau)}|x(s)|^{2} d s\right] \\
& \leq E \int_{-\tau}^{0} e^{\varepsilon \tau}|x(s)|^{2} d s, \\
& E \int_{0}^{t} e^{\varepsilon s}\left[\int_{-\infty}^{0}|x(s+\theta)|^{2} d \mu(\theta)-\mu_{r}|x(s)|^{2}\right] d s \\
& =E\left\{\int _ { 0 } ^ { t } e ^ { \varepsilon s } \left[\int_{-\infty}^{-s} e^{2 r(s+\theta)}|x(s+\theta)|^{2}\right.\right. \\
& \left.\times e^{-2 r(s+\theta)} d \mu(\theta)\right] d s \\
& +\int_{0}^{t} e^{\varepsilon s} \int_{-s}^{0}|x(s+\theta)|^{2} d \mu(\theta) d s \\
& \left.-\mu_{r} \int_{0}^{t} e^{\varepsilon s}|x(s)|^{2} d s\right\} \\
& \leq E\left\{\|\xi\|_{C_{r}}^{2} \int_{0}^{t} e^{(\varepsilon-2 r) s} d s \int_{-\infty}^{0} e^{-2 r \theta} d \mu(\theta)\right. \\
& +\int_{-t}^{0} \int_{-\theta}^{t} e^{\varepsilon s}|x(s+\theta)|^{2} d s d \mu(\theta) \\
& \left.-\mu_{r} \int_{0}^{t} e^{\varepsilon s}|x(s)|^{2} d s\right\} \\
& \leq E\left\{\|\xi\|_{C_{r}}^{2} \mu_{r} \int_{0}^{t} e^{(\varepsilon-2 r) s} d s\right. \\
& +\int_{-\infty}^{0} \int_{0}^{t} e^{\varepsilon(s-\theta)}|x(s)|^{2} d s d \mu(\theta) \\
& \left.-\mu_{r} \int_{0}^{t} e^{\varepsilon s}|x(s)|^{2} d s\right\} \\
& \leq E\left\{\|\xi\|_{C_{r}}^{2} \mu_{r} t+\int_{-\infty}^{0} e^{-\varepsilon \theta} d \mu(\theta)\right. \\
& \times \int_{0}^{t} e^{\varepsilon s}|x(s)|^{2} d s \\
& \left.-\mu_{r} \int_{0}^{t} e^{\varepsilon s}|x(s)|^{2} d s\right\} \\
& \leq E\|\xi\|_{C_{r}}^{2} \mu_{r} t \text {. }
\end{aligned}
$$

Hence, we derive

$$
\begin{aligned}
e^{\varepsilon t} E V_{4}(x(t)) \leq & E V_{4}(\xi(0))+\frac{K_{3} e^{\varepsilon t}}{\varepsilon} \\
& +\frac{n p}{2}\left(E \mu_{r}\|\xi\|_{C_{r}}^{2} t+E \int_{-\tau}^{0} e^{\varepsilon \tau}|x(s)|^{2} d s\right) .
\end{aligned}
$$

This implies that $\lim \sup _{t \rightarrow \infty} E V_{4}(x(t)) \leq \varepsilon^{-1} K_{3}$. 
Again, by the fact that $|x|^{2} \leq n \max _{1 \leq i \leq n} x_{i}^{2}$, it then follows that $|x|^{p} \leq n^{p / 2} \max _{1 \leq i \leq n} x_{i}^{p} \leq n^{p / 2} V_{4}(x)$, and we have

$$
\limsup _{t \rightarrow \infty} E|x(t)|^{p} \leq G(p) ;
$$

the assertion follows by setting $G=G(p)=n^{p / 2} \varepsilon^{-1} K_{3}$. The proof is complete.

Remark A. If (H1) and (H2) are valid, then $\limsup _{t \rightarrow \infty} P\{|x(t)| \leq \chi\} \geq 1-\varepsilon$; that is, solution $x(t)$ to model (2) is stochastically ultimately bounded.

If we take $p=1 / 2$, then the result is valid by using of Theorem 4 and Chebyshev's inequality. The proof is omitted herewith.

Theorem 5. If (H1) and (H2) hold, there exists a positive constant $Q$, and then

$$
\limsup _{T \rightarrow \infty} \frac{1}{T} \int_{0}^{T} D\left(x_{i}(t)\right) d t \leq Q, \quad i=1,2, \ldots, n .
$$

Proof. Rewrite (20) as

$$
\begin{aligned}
H(x)= & H_{1}(x)-(n p+1)|x|^{2} \\
& +\frac{n p}{2}\left(e^{\varepsilon \tau}+\mu_{r}\right)|x|^{2}+\varepsilon V_{4}(x),
\end{aligned}
$$

with

$$
\begin{aligned}
H_{1}(x)= & \sum_{i=1}^{n} p h_{i u} x_{i}^{p}+\frac{p}{2} \sum_{i=1}^{n} \sum_{j=1}^{n}\left(\left|a_{i j}\right|_{u}+\left|b_{i j}\right|_{u}^{2}+\left|c_{i j}\right|_{u}^{2}\right) x_{i}^{2 p} \\
& +\frac{p}{2} \sum_{i=1}^{n} \sum_{j=1}^{n}\left|a_{i j}\right|_{u} x_{j}^{2}+(n p+1)|x|^{2} \\
& -\frac{p(1-p)}{2} \sum_{i=1}^{n} K_{2} x_{i}^{p}\left(x_{i}-x_{i}^{*}\right)^{2} .
\end{aligned}
$$

Clearly, $H_{1}(x)$ is bounded in $R_{++}^{n}$; namely, $K_{4}$ := $\sup _{x \in R_{++}^{n}} H_{1}(x)<\infty$. So

$$
H(x) \leq K_{4}-(n p+1)|x|^{2}+\frac{n p}{2}\left(e^{\varepsilon \tau}+\mu_{r}\right)|x|^{2}+\varepsilon V_{4}(x) .
$$

By (19), we have

$$
\begin{aligned}
d V_{4}(x(t)) \leq & \left\{K_{4}-(n p+1)|x(t)|^{2}\right. \\
& +\frac{n p}{2}\left[|x(t-\delta(t))|^{2}\right. \\
& \left.\left.\quad+\int_{-\infty}^{0}|x(t+\theta)|^{2} d \mu(\theta)\right]\right\} d t \\
& +p \sum_{i=1}^{n} x_{i}^{p}(t) g_{i} d B_{i}(t) .
\end{aligned}
$$

Integrating both sides of (29) from 0 to $T$ ( $T>0$ is arbitrary) and then taking expectations, we obtain that

$$
\begin{aligned}
E\left(V_{4}(x(T))\right) \leq & V_{4}(\xi(0))+K_{4} T \\
& -E \int_{0}^{T}|x(t)|^{2} d t+\frac{n p}{2} I_{1}+\frac{n p}{2} I_{2} .
\end{aligned}
$$

Similar to (22), by the definition of $\delta(\cdot)$, we can derive

$$
\begin{gathered}
I_{1}=E \int_{0}^{T}\left(|x(t-\delta(t))|^{2}-|x(t)|^{2}\right) d t \leq \int_{-\tau}^{0}|x(t)|^{2} d t, \\
I_{2}=E \int_{0}^{T}\left[\int_{-\infty}^{0}|x(t+\theta)|^{2} d \mu(\theta)-|x(t)|^{2}\right] d t \leq \frac{1}{2 r} \mu_{r}\|\xi\|_{C_{r}}^{2} .
\end{gathered}
$$

Substituting (31) into (30), then we have

$$
\limsup _{T \rightarrow \infty} \frac{1}{T} \int_{0}^{T} E|x(t)|^{2} d t \leq K_{4}:=Q
$$

By the fact that $D\left(x_{i}\right)=E\left|x_{i}\right|^{2}-\left(E x_{i}\right)^{2}$ and $\lim _{t \rightarrow \infty} E(x(t))=$ $x^{*}$, the conclusion is obviously valid. The proof is complete.

\section{Example and Numerical Simulation}

Now, we will simulate asymptotic behaviors of solutions to model (2). Let us consider the following 1-dimensional stochastic autonomous Logistic model:

$$
\begin{aligned}
& d x(t)=x(t)[h-a x(t)-b x(t-\tau) \\
& \left.-c \int_{-\infty}^{0} x(t+\theta) d \mu(\theta)\right] d t \\
& +x(t)\left[\sigma\left(x(t)-x^{*}\right)+\alpha x(t)\left(x(t)-x^{*}\right)\right. \\
& +\beta x(t-\tau)\left(x(t)-x^{*}\right) \\
& +\gamma \int_{-\infty}^{0} x(t+\theta) d \mu(\theta) \\
& \left.\times\left(x(t)-x^{*}\right)\right] d B(t),
\end{aligned}
$$

with initial data $\xi(\theta) \in C_{r}$, where $h, a, b, c, \tau, \sigma, \alpha, \beta$, and $\gamma$ are all positive numbers, and the equilibrium state is $x^{*}=h /(a+b+c)$. By Theorem 2, if $a>0.5 x^{*}\left(\sigma^{2}+K \alpha^{2}+\right.$ $\left.K \beta^{2}+K \gamma^{2}\right)$, then the equilibrium state $x^{*}$ of model (33) is globally asymptotically stable a.s.. According to Milstein method mentioned in Higham [11], the initial data of model 
(33) is given by $\xi(\theta)=0.32 e^{0.5 \theta}$ and $\mu(\theta)=e^{\theta}, \theta \in(-\infty, 0]$; the difference equation is followed next

$$
\begin{aligned}
x^{(k+1)}-x^{(k)}= & x^{(k)}\left[h-a x^{(k)}-b x^{(k-\tau / \Delta t)}\right. \\
& \left.-\frac{2 c}{3} e^{-k \Delta t}-c e^{-k \Delta t} \sum_{i=1}^{k} x^{(i)} e^{i \Delta t} \Delta t\right] \Delta t \\
& +\sigma x^{(k)}\left(x^{(k)}-x^{*}\right) \sqrt{\Delta t} \xi^{(k)} \\
& +\frac{\sigma^{2}}{2} x^{(k)}\left(x^{(k)}-x^{*}\right)\left(\left(\xi^{(k)}\right)^{2}-1\right) \Delta t \\
& +\alpha x^{(k)} x^{(k)}\left(x^{(k)}-x^{*}\right) \sqrt{\Delta t} \xi^{(k)} \\
& +\frac{\alpha^{2}}{2} x^{(k)} x^{(k)}\left(x^{(k)}-x^{*}\right)\left(\left(\xi^{(k)}\right)^{2}-1\right) \Delta t \\
& +\beta x^{(k)} x^{(k-\tau / \Delta t)}\left(x^{(k)}-x^{*}\right) \sqrt{\Delta t} \xi^{(k)} \\
& +\frac{\beta^{2}}{2} x^{(k)} x^{(k-\tau / \Delta t)}\left(x^{(k)}-x^{*}\right)\left(\left(\xi^{(k)}\right)^{2}-1\right) \Delta t \\
& \left.+\frac{\gamma^{2}}{2}\left(\frac{2 c}{3} e^{-k \Delta t}+c e^{-k \Delta t} \sum_{i=1}^{k} x^{(i)} e^{i \Delta t} \Delta t\right) x^{(k)}-x^{*}\right)\left(\left(\xi^{(k)}\right)^{2}-1\right) \Delta t, \\
& +\gamma\left(\frac{2 c}{3} e^{-k \Delta t}+c e^{-k \Delta t} \sum_{i=1}^{(i)} e^{i \Delta t} \Delta t\right) x^{(k)} \\
& \left(x^{(k)}-x^{*}\right) \sqrt{\Delta t} \xi^{(k)} \\
& \\
& \\
& \\
& \\
& \\
&
\end{aligned}
$$

where $\xi^{(k)}(k=1,2, \ldots, n)$ is the Gaussian random variable which follows the standard normal distribution $\mathcal{N}(0,1)$. If we fix the parameters $h=0.5, a=0.6, b=0.1, c=0.3, \tau=1$, and $\Delta t=0.01$, then $x^{*}=h /(a+b+c)=0.5$. When $\sigma=$ $\alpha=\beta=\gamma=0$, model (33) becomes a deterministic one; then the equilibrium state $x^{*}$ is globally asymptotically stable a.s. by Theorem 2 (see Figure 1). When $\sigma=0.1$ and $\alpha=\beta=$ $\gamma=0$, the intrinsic growth rate of model (33) is perturbed by white noise; if $a>0.5 x^{*} \sigma^{2}+b+c$, the equilibrium state $x^{*}$ is globally asymptotically stable a.s. (see Figure 2 ). When $\sigma=0.1$ and $\alpha=\beta=\gamma=0.01$, that is, each parameter of model (33) is fluctuated by white noise, if $a>0.5 x^{*}\left(\sigma^{2}+\right.$ $\left.K \alpha^{2}+K \beta^{2}+K \gamma^{2}\right)+b+c$ and $K=\left(0.32 e^{(r+0.5) \theta}+x^{(k)}\right)^{2} \leq$ $\left(0.32+x^{(k)}\right)^{2}$ are satisfied, then the equilibrium stable $x^{*}$ is globally asymptotically stable a.s. (see Figure 3 ).

\section{Appendix}

From the definition of $V_{2}(t)$, we obtain

$$
d V_{2}(x(t)) \leq F(x(t)) d t+\sum_{i=1}^{n} p\left(x_{i}^{p}(t)-1\right) g_{i} d B_{i}(t),
$$

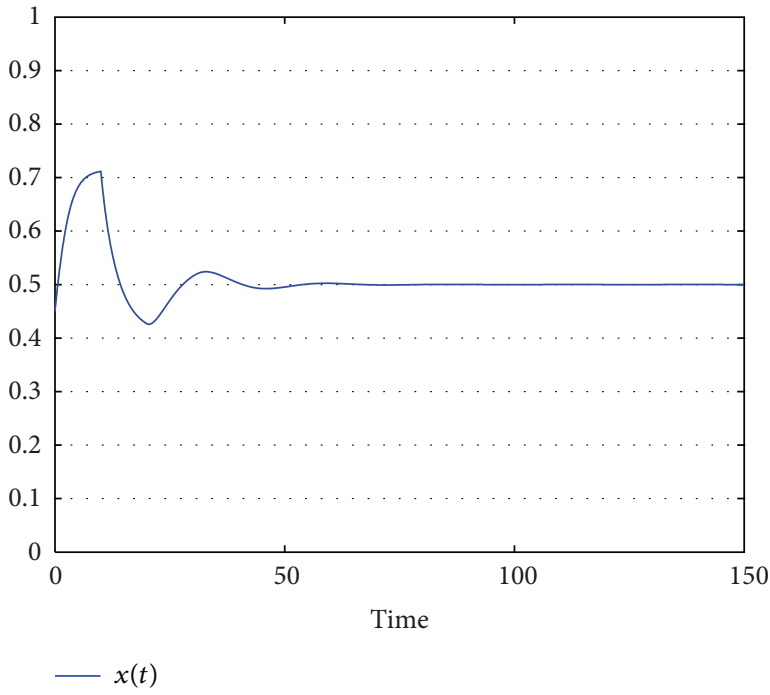

Figure 1: Model (33) becomes a deterministic model.

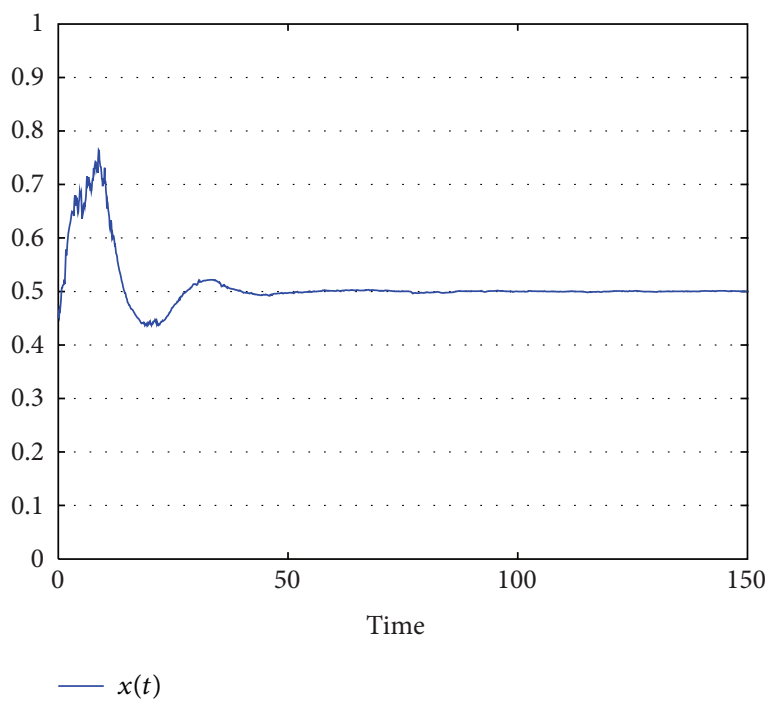

FIGURE 2: The intrinsic growth rate of model (33) is perturbed by white noise.

where

$$
\begin{aligned}
F(x)=\sum_{i=1}^{n}\{ & p h_{i u}\left(x_{i}^{p}+1\right) \\
& +\frac{p}{2} \sum_{j=1}^{n}\left(\left|a_{i j}\right|_{u}+\left|b_{i j}\right|_{u}^{2}+\left|c_{i j}\right|_{u}^{2}\right)\left(x_{i}^{p}-1\right)^{2} \\
& +\frac{p}{2} \sum_{j=1}^{n}\left(\left|a_{i j}\right|_{u}+2\right) x_{j}^{2}+\frac{p K_{1}}{2}\left(x_{i}-x_{i}^{*}\right)^{2} \\
& \left.-\frac{p(1-p) K_{2}}{2} x_{i}^{p}\left(x_{i}-x_{i}^{*}\right)^{2}\right\} .
\end{aligned}
$$




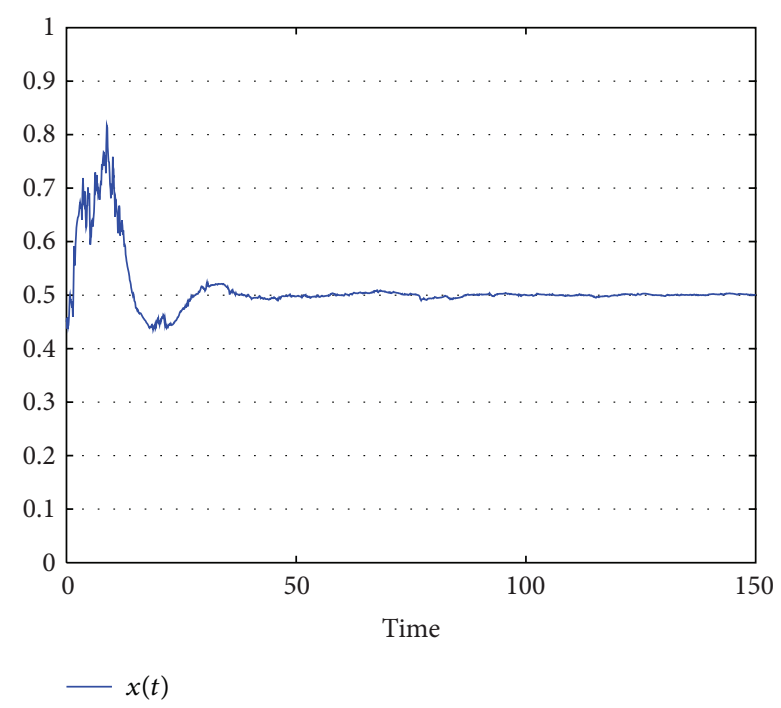

FIGURE 3: Each parameter of model (33) is fluctuated by white noise.

It is easy to check that $F(x) \leq K^{\prime}<\infty$. Equation (A.1) becomes

$$
d V_{2}(x(t)) \leq K^{\prime} d t+\sum_{i=1}^{n} p\left(x_{i}^{p}(t)-1\right) g_{i} d B_{i}(t) .
$$

Integrating both sides of (A.3) from 0 to $\tau_{k} \wedge T$ ( $T>0$ is arbitrary) and then taking expectations, it yields that

$$
E V_{1}\left(x\left(\tau_{k} \wedge T\right)\right) \leq E V_{2}\left(x\left(\tau_{k} \wedge T\right)\right) \leq V_{2}(\xi(0))+K^{\prime} T .
$$

Noting that for every $w \in \Omega_{k}=\left\{\tau_{k} \leq T\right\}$, by the definition of stopping time $\tau_{k}, x_{i}\left(\tau_{k}, w\right)=k$ or $1 / k$ for some $i=1,2, \ldots, n$, $V_{1}\left(x\left(\tau_{k} \wedge T\right)\right) \geq \min \left\{k^{p}-p \log k, k^{-p}+p \log k\right\}$. It then follows that

$$
\begin{aligned}
& P\left\{\tau_{k} \leq T\right\}\left(k^{p}-p \log k\right) \wedge\left(k^{-p}+p \log k\right) \\
& \quad \leq E\left[I_{\left\{\tau_{k} \leq T\right\}}(w) V_{2}\left(x\left(\tau_{k}, w\right)\right)\right] \\
& \quad \leq V_{2}(\xi(0))+K^{\prime} T .
\end{aligned}
$$

Thus, $\lim _{k \rightarrow \infty} P\left\{\tau_{k} \leq T\right\}=0$. Since $T>0$ is arbitrary, we must have $P\left\{\tau_{\infty}<\infty\right\}=0$; then $P\left\{\tau_{\infty}=\infty\right\}=1$ as required.

\section{Conflict of Interests}

The authors declare that there is no conflict of interests regarding the publication of this paper.

\section{Acknowledgments}

This work is supported by the Natural Science Foundation of China (no. 11201075), the Natural Science Foundation of Fujian Province of China (no. 2010J01005), and the Technology Innovation Platform Project of Fujian Province (no. 2009J1007).

\section{References}

[1] A. Bahar and X. Mao, "Stochastic delay Lotka-Volterra model," Journal of Mathematical Analysis and Applications, vol. 292, no. 2, pp. 364-380, 2004.

[2] X. Mao, "Delay population dynamics and environmental noise," Stochastics and Dynamics, vol. 5, no. 2, pp. 149-162, 2005.

[3] D. Jiang and N. Shi, "A note on nonautonomous logistic equation with random perturbation," Journal of Mathematical Analysis and Applications, vol. 303, no. 1, pp. 164-172, 2005.

[4] Y. Shen, G. Zhao, M. Jiang, and X. Mao, "Stochastic LotkaVolterra competitive systems with variable delay," in Advances in Intelligent Computing, vol. 3645 of Lecture Note in Computer Science, pp. 238-247, 2005.

[5] M. Liu and K. Wang, "Global asymptotic stability of a stochastic Lotka-Volterra model with infinite delays," Communications in Nonlinear Science and Numerical Simulation, vol. 17, no. 8, pp. 3115-3123, 2012.

[6] Y. Huang, Q. Liu, and Y. Liu, "Global asymptotic stability of a general stochastic Lotka-Volterra system with delays," Applied Mathematics Letters, vol. 26, no. 1, pp. 175-178, 2013.

[7] Y. Xu, F. Wu, and Y. Tan, "Stochastic Lotka-Volterra system with infinite delay," Journal of Computational and Applied Mathematics, vol. 232, no. 2, pp. 472-480, 2009.

[8] Y. Xu, Stochastic Differential Equations with Infinite Delay and Applications, Huazhong University of Science and Technology, Wuhan, China, 2009.

[9] J. Hale and J. Kato, "Phase space for retarded equations with infinite delay," Funkcialaj Ekvacioj, vol. 21, no. 1, pp. 11-41, 1978.

[10] F. Wei, The Basic Theory of Stochastic Functional Differential Equations with Infinite Delay, Northeast Normal University, Changchun, China, 2006.

[11] D. Higham, "An algorithmic introduction to numerical simulation of stochastic differential equations," SIAM Review, vol. 43, no. 3, pp. 525-546, 2001. 


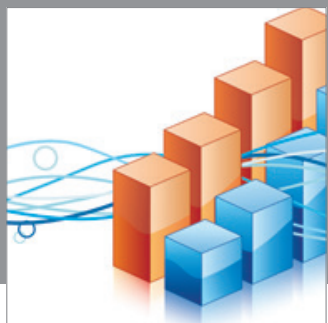

Advances in

Operations Research

mansans

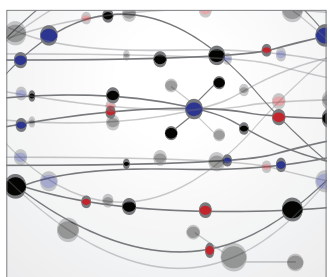

The Scientific World Journal
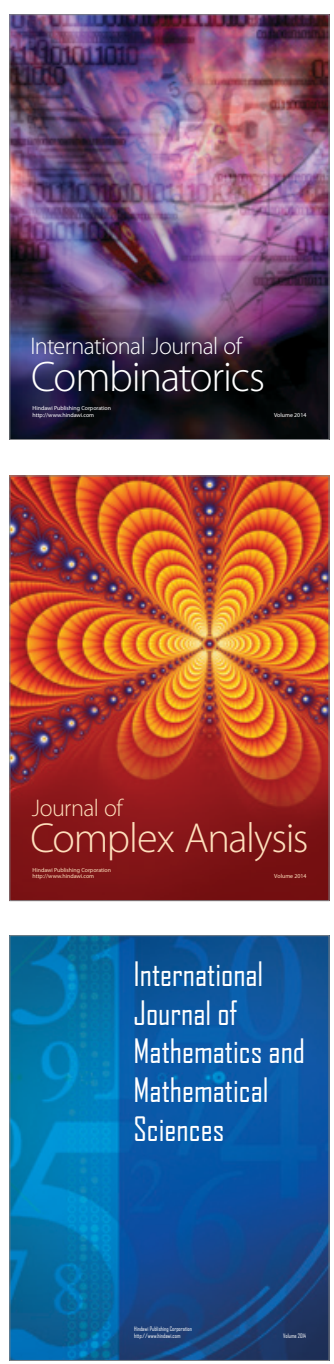
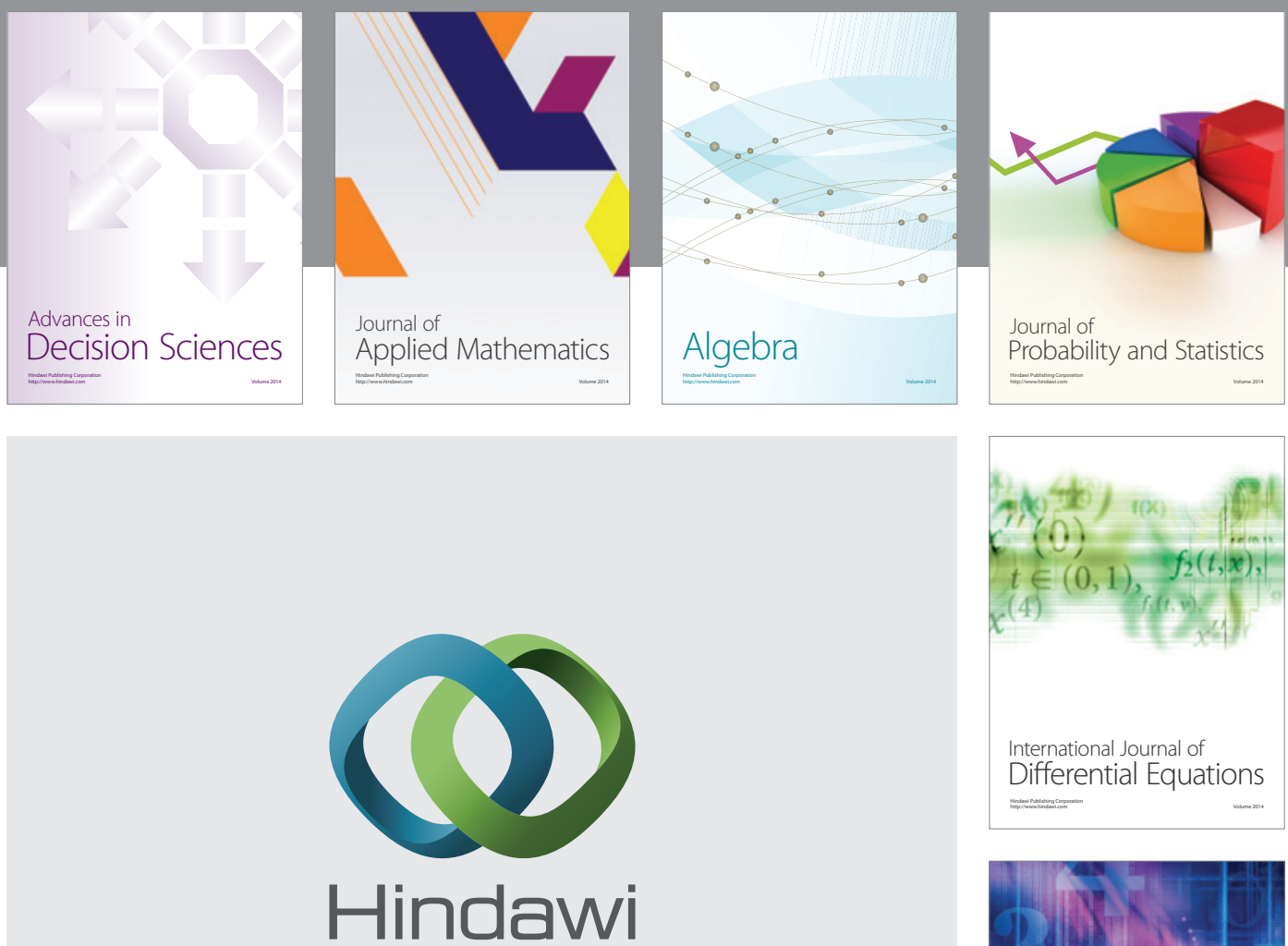

Submit your manuscripts at http://www.hindawi.com
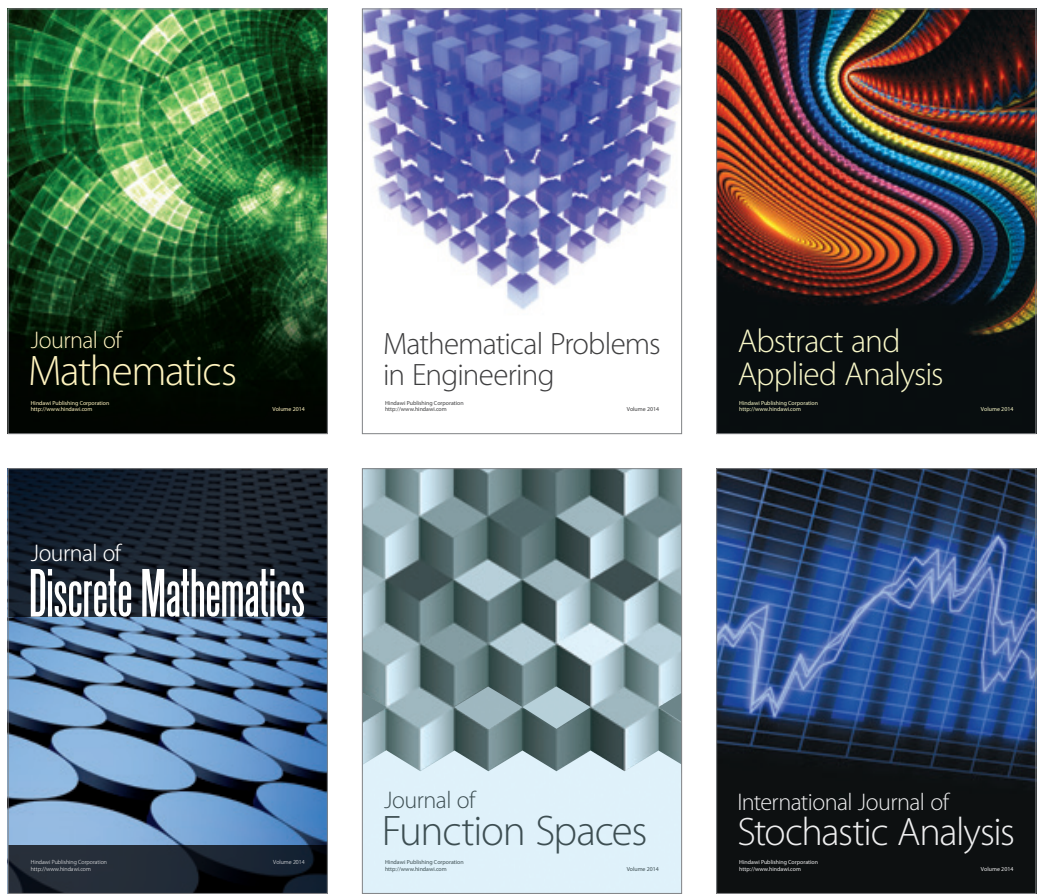

Journal of

Function Spaces

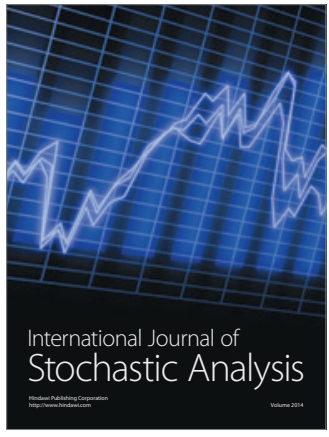

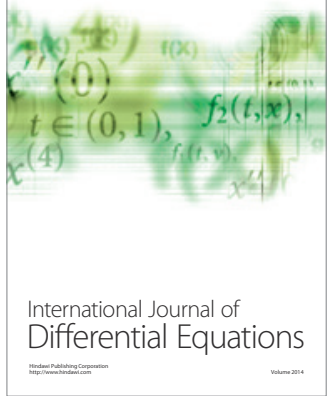
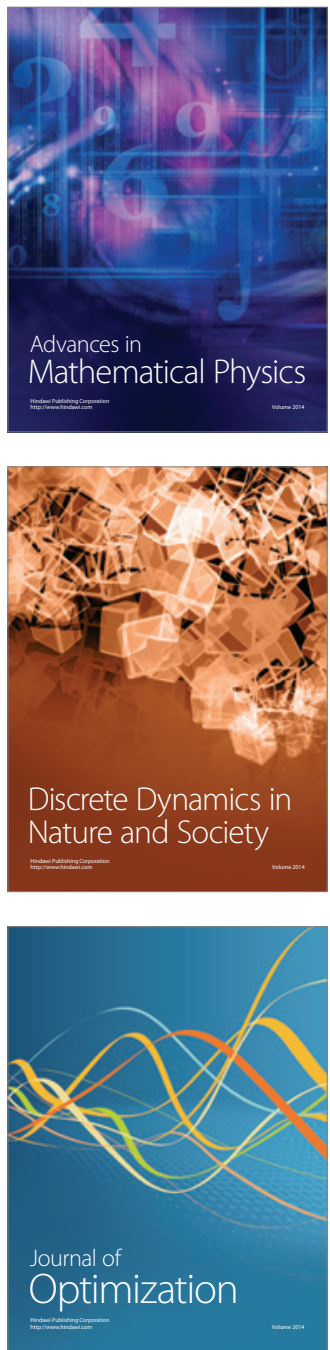